\title{
Oscillations in main sequence B-type stars - challenges to theory
}

\author{
W. A. Dziembowski \\ Warsaw University Observatory and Copernicus Astronomical Center, Warsaw, Poland
}

\begin{abstract}
The current status of our understanding of the diversity of B-star pulsation is presented with an emphasis on unsolved problems. Not all detected modes are found unstable in standard models. The proposed way of extending the instability by invoking an iron accumulation in the driving zone is not free of difficulties. There are still controversies regarding the excitation of slow modes in Be stars. Nonlinear modelling of radial pulsations in $\beta$ Cephei stars results in much higher amplitudes than observed. There must be hidden modes involved but we may only speculate about their nature.
\end{abstract}

\section{Introduction}

Not long after the driving mechanism for pulsation in stars of the Cepheid instability strip has been identified, efforts were made to explain the origin of pulsations in $\beta$ Cephei stars. For about two decades this constituted the greatest challenge to stellar pulsation theory. While theorists were still searching the explanation for $\beta$ Cep pulsation, new types of periodic B-star variability were discovered by observers. Interestingly, the new variables, though less luminous than $\beta$ Cep stars, had longer periods. The first such objects were discovered by Smith (1977) by means of spectroscopy. These were rapid rotators and their variability was detected in line profiles. The term Slowly Pulsating B-type (SPB) stars denoting all mid to late B-type long period variables was defined 14 years later by Waelkens (1991), who discovered many such objects in his photometric survey. These discoveries added a new problem to the long-standing challenge of explaining the origin of hot star pulsation.

The answer that came in the early 1990ties thanks to sophisticated calculations of stellar opacities by the OPAL team (Iglesias et al. 1992) was not unexpected. In fact, Simon's (1982) call for a revision of stellar opacity data was motivated by the problem of the driving mechanism for $\beta$ Cep pulsation. Shortly after the OPAL opacities became available for stellar modelling, the first papers demonstrating that there are unstable modes in B stars with periods consistent with observations were published (Cox et al. 1992; Kiriakidis et al. 1992; Moskalik \& Dziembowski 1992; Dziembowski \& Pamyatnykh 1993; Gautschy \& Saio 1993; Dziembowski et al. 1993). In the latter three papers the instability of high order g-modes responsible for slow pulsation was demonstrated. The puzzle was solved. The driving effect is caused by the classical $\kappa$-mechanism acting in the layer of the local opacity maximum arising primarily due to the line opacities of ionized iron. This maximum occurs at a temperature of about $2 \times 10^{5} \mathrm{~K}$ and is often referred to as the Fe-bump or Z-bump.

The identification of the driving mechanism did not stop the interest in B-star pulsation. In recent years, the main effort was focused on applications of seismic sounding, which required collecting more data on the excited modes. There have been fruitful ground-based campaigns on selected $\beta$ Cep stars. Very interesting data on various B-type pulsators were obtained with the MOST space telescope. The new data not only led to valuable constraints on stellar models and internal rotation but also revealed that our understanding of B-star pulsation is by far not satisfactory. 


\section{The opacity mechanism in B stars}

The occurrence of two distinct types of B-type pulsators is a consequence of the existence of two distinct types of modes which have large amplitudes in the Z-bump layer and have periods matching the thermal relaxation time of that layer. With these two conditions satisfied, the driving effect may be strong enough to overcome the damping occurring outside this layer.

The first type encompasses $\mathrm{p}$ - and g-modes of the lowest radial orders $n$ and angular degrees $\ell$. In the outer layers, the properties of such oscillations are determined by the frequency $\omega$, and are nearly independent of $\ell$. These modes are unstable in somewhat evolved main sequence stars of spectral types earlier than B4. The simultaneous excitation of $p$ - and g-modes is important for seismic diagnoses because their frequencies are sensitive to the deep interior stellar structure. The instability extends to high- $\ell$ modes trapped in the envelope.

The second type of B star pulsations encompasses g-modes of high orders. The instability range depends on the value of $\ell(\ell+1) / \omega^{2}$ which determines the depth dependence of the pulsation amplitude and separately on $\omega$, which determines the time-scale matching. At low $\ell$, the period match occurs for objects of lower masses and later spectral types than $\beta$ Cep stars. This explains the occurrence of the SPB domain in the main sequence band. The instability of the high- $n$ modes occurs in wider ranges of stellar parameters. However, in models of more massive stars it was originally found only for high- $\ell$ modes, which are not easily detectable in light variations. Thus, we have thought that the $\beta$ Cep-type and SPB-type pulsation are mutually exclusive. Observations taught us that we have been wrong.

The driving mechanism in all B-type pulsators is the same. It is the $\kappa$-mechanism in its cleanest form and there is no role for convection. This is not true for main sequence F-type stars. This is why the slowly pulsating $\gamma$ Doradus stars cannot be regarded as an SPB star analog. The role of convection in the former stars is essential, while it is only secondary in the somewhat brighter and much more rapidly pulsating $\delta$ Scuti stars. True analogs of the two types of main sequence B-type pulsators were found among the sdB stars and were explained with the same driving mechanism (Fontaine et al. 2003).

\section{Does iron accumulate in the driving zone of $\beta$ Cephei stars?}

$\nu$ Eridani is perhaps the most thoroughly studied $\beta$ Cephei star. Recent observational campaigns (Aerts et al. 2004, Handler et al. 2004, Jerzykiewicz et al. 2005) on this object have been very rewarding. New modes were found and for most of them unique identifications were found (De Ridder et al. 2004). The data were used for seismic model construction which led to implications regarding the global parameters of the star, internal mixing and rotation (Pamyatnykh et al. 2004, Ausseloos et al. 2004) and stellar opacities (Daszyńska-Daszkiewicz et al. 2005). However, the models were not totally successful as they predict mode instability only in the 4 to $6 \mathrm{c} / \mathrm{d}$ range while in $\nu$ Eri's oscillation spectrum there is a number of peaks in the 6 to $8 \mathrm{c} / \mathrm{d}$ range and a peak at $0.42 \mathrm{c} / \mathrm{d}$. The explanation proposed by Pamyatnykh et al. (2004) was that the iron abundance in the driving layer is significantly enhanced due to selective radiation pressure. The required enhancement to destabilize high frequency modes was somewhat less than a factor of four and somewhat larger for the low-frequency mode. The proposal was based on Charpinet et al.'s (1996) solution of the driving problem for the sdB pulsators, but it was not clear then whether or not this applies because of huge difference in properties between the two types of objects.

Detailed calculations of element segregation due to diffusion and radiative pressure for main sequence B-stars were made by Seaton (1999), but only for stars with a mass up to $4.5 M_{\odot}$, which is about half of $\nu$ Eri's mass. These calculations, which were made assuming no microscopic mixing, showed that a significant iron accumulation in the Z-bump may indeed take place. However, the enhancement should occur also in the atmosphere and should thus be visible. Calculations for a model appropriate for $\nu$ Eri (Bourge et al. 2007) show 
a higher iron enhancement in the atmosphere which is consistent with the trend seen in Seaton's results. In main sequence stars with masses less than $3 M_{\odot}$ and in $\mathrm{sdB}$ stars, the iron enhancement may be hidden because there is a region between the Z-bump layer and the photosphere where gravitational settling of iron dominates over selective radiation pressure. Since there is no spectroscopic evidence for iron enhancement in $\nu$ Eri, the proposed solution seems invalidated. Paradoxically, as Turcotte and Richard (2005) pointed out, none of the $\mathrm{HgMn}$ stars - the only B stars showing evidence of diffusive element segregation - is known as a pulsator. Another problem is the neglect of rotational mixing. In the case of $\nu$ Eri it might be justified because of its unusually slow equatorial velocity of some $6 \mathrm{~km} / \mathrm{s}$. However, this is not the only object, which is a hybrid $\beta$ Cep/SPB pulsator. The oscillation spectrum of 12 Lac (Handler et al. 2006) is strikingly similar, but its rotational velocity is much higher.

\section{How rotation affects slow modes}

The nature of the variability with periods in the range from one half to a few days observed in Be stars has been a subject of controversy. The interpretation in terms of slow modes, first proposed by Baade (1982), is partially supported by linear stability calculations. On the other hand, Balona (1995) argues that the observed periodic light variability may be explained by spots on the rotating stellar surface. The ambiguity is caused by the fact that the rotation periods of $\mathrm{Be}$ stars and the periods of unstable modes are similar. This coincidence implies that rotation cannot be regarded as a small perturbation and that the angular dependence of the modes cannot be described by individual spherical harmonics.

Two approaches have been adopted to treat the large effects of rotation. One developed by Lee and Saio (1989) relies on a truncated expansion of the longitudinal mode dependence into Legendre functions. The other, which relies on the traditional approximation, allowing for a separation of the angular dependence in terms of the Hough functions, has been used for instance by Townsend (2005) in his extensive survey of slow-mode instability in B-stars. The traditional approximation greatly facilitates the stability analysis because the effect of rotation essentially reduces to replacing $\ell(\ell+1)$ with a $\lambda$ parameter, which for specified angular orders depends only on $\Omega / \omega$ - the ratio of angular velocity rotation to mode frequency. Townsend (2005) showed that rotation has only a small effect on the extent of the SPB domain in the HR diagram. The effect on the frequency range of unstable modes in the inertial system and on mode visibility may be much more significant.

The oscillation spectrum of the Be star HD 163868, determined from MOST data by Walker et al. (2005), is far the richest for an object of this type. The spectrum shows three abundant groups of peaks: one below $0.5 \mathrm{c} / \mathrm{d}$, one centred near $1.5 \mathrm{c} / \mathrm{d}$, and one near $3 \mathrm{c} / \mathrm{d}$. The authors could explain the two latter groups in terms of unstable modes in an appropriate stellar model. Only for the highest-frequency peaks of the first group they could find counterparts among unstable modes. Repeating the stability analysis for the same model but using the traditional approximation, we (Dziembowski et al. 2007) obtained a somewhat different result. In particular, we had no difficulty in explaining all low frequency peaks in terms of unstable retrograde g-modes of the lowest angular order and we could account for mode selection taking into account the visibility dependence on the aspect and assuming $i \approx 90^{\circ}$. With $i \approx 55^{\circ}$, as adopted by Walker et al. (2005), we could not obtain any consistent interpretation of the observed oscillation spectrum.

\section{Beyond linear theory}

Linear stability calculations provide only a partial answer to the question which modes may be excited and do not predict pulsation amplitudes. From observations, we know that the typical form of B-star pulsation consists of the excitation of many modes with low amplitudes. 
There are only three $\beta$ Cep stars with a $\mathrm{V}$ amplitude exceeding 0.08 mag. Surprisingly, two of the three stars were discovered only very recently (Pigulski 2007). Typical amplitudes of known modes in B-type pulsators are much lower. Why this form of pulsation is chosen may be answered only by going beyond linear theory.

Recent nonlinear modelling of radial pulsations of $\beta$ Cep stars by Smolec and Moskalik (2007) predicts light amplitudes that are significantly higher than even in these three highamplitude and apparently single-mode pulsators. These authors conclude that there must be hidden modes involved in the pulsations of these stars. Indeed, their results suggest that saturation of the instability by a single-mode is unlikely. Although in most cases they find single-mode pulsation as the limit cycle, they also find cases of sustained double mode (DM) pulsation involving the fundamental and first overtone modes. The DM pulsation occurred either due to properties of saturation (non-resonant DM pulsation) or due to the resonance $2 \omega_{\text {lov }} \approx \omega_{\text {fund }}+\omega_{\text {llov }}$. The first two involved modes were linearly unstable, while the second overtone is stable.

What was found rather rare in models that know only about radial modes should be common in a realistic situation. The non-resonant DM behaviour found by Smolec and Moskalik (2007) occurs in the intermediate $T_{\text {eff-range between the first overtone and fundamental }}$ mode domains. Since modes differing in $\ell$ in narrow frequency ranges have similar saturation coefficients, we expect that the domain of stable single-mode pulsation may totally disappear. Moreover, there are many more possibilities for resonant coupling. At the lowest order in amplitude of radial modes there is a possibility of parametric excitation of nonradial modes with the same $\ell$ and opposite $m$ values with frequencies whose sum is close to the radial mode frequency, $\omega$. If the sum is close to $2 \omega$, then the parametric excitation may occur due to a higher order effect, similar to that responsible for the resonant DM pulsation found by Smolec and Moskalik (2007). The effect requires higher amplitude, but nonlinear coupling is stronger between modes of higher frequencies, which all have large amplitude in the outer envelope.

Amplitude growth may be terminated in two distinct ways. One is through induced changes in the mean stellar structure and the other is through a resonant excitation of stable (parasite) modes. In the first case, the discrepancy could be blamed to the presence of undetected nonradial modes. Especially those of high $\ell$ may indeed contribute to saturation and have disc-averaged light amplitudes below the detection threshold. In the absence of resonance, the lowest order equations for the evolution of mode amplitudes may be written in the form

$$
\frac{d A_{j}}{d t}=\gamma_{j}\left(1-\sum_{k=1}^{K} \alpha_{j k} A_{k}^{2}\right),
$$

where $A_{j}$ denotes surface-averaged amplitudes, $\gamma_{j}$ the linear growth rates, and $\alpha_{j k}$ are introduced ad hoc saturation coefficients, which are assumed greater than zero. For $A_{j}$ we may choose the r.m.s. value of $\delta R / R$ but as long as $\alpha_{j k}$ remains unspecified the choice does not matter. Around each radial mode, there are always many unstable nonradial modes of similar frequencies and, hence, similar saturation coefficients. At a steady limit cycle we should have $\sum_{k=1}^{K} \alpha_{j k} A_{k}^{2}=1$. Compared with the single-mode case, we may expect, crudely, a factor $K^{-1 / 2}$ reduction of the r.m.s. amplitude of individual modes. If the $\ell$-values of the excited modes are larger than 2 , the modes most likely remain undetectable in light variations. Amplitudes may be further reduced if high-order g-modes are excited. Such modes are unstable in $\beta$ Cep stars in a certain range of $\ell$ values but we cannot assume for them similar saturation coefficients as for p-modes.

If we assume that only p-modes take part in the saturation of the instability, then we may rule out this effect as the sole cause of amplitude limitation. If this were the case, then, assuming again that excited modes have similar frequencies, the r.m.s. velocity fluctuation should be of the same order as the radial velocity amplitude determined by Smolec and 
Moskalik (2007) for radial pulsation. These authors found values up to $450 \mathrm{~km} / \mathrm{s}$. Thus, if the undetected modes are responsible for most of the instability saturation in $\beta$ Cep stars, we should expect line broadening corresponding to macroturbulence at a level of $100 \mathrm{~km} / \mathrm{s}$, which is not observed. We must conclude that the instability in $\beta$ Cep stars is not saturated by $\mathrm{p}$-modes. Still, we should consider a possible role of unstable high-order g-modes. Since no nonlinear calculations have been done on such modes, we have to rely on a guess, which seems reasonable, that the mode contribution to saturation is determined by the amplitude of $\delta T_{\text {eff }}$. Adopting a linear relation between $\delta T_{\text {eff }}$ and the pulsation velocity component, we may compare the r.m.s. velocities at saturation by g- and p-modes. My linear calculations showed that in the g-mode case the velocities are by factors between three and four lower, but still too high. Thus, it seems that the instability is not saturated and that the excess of work is consumed by parasite modes.

\section{Challenges that remain}

The puzzle of B-star pulsation has been solved at the beginning of the 1990ties. The classical opacity mechanism was shown to drive the whole variety of modes seen in B stars. New data that came after, though not shaking our basic understanding of the driving effect, point to a need for further work on better understanding pulsation in these stars. This is timely because if we do not understand an important aspect of stellar pulsation it means that we do not understand something important in stellar physics.

There are problems on the level of linear theory. Not all modes that are detected are found unstable. With present microscopic physics data it seems impossible to explain the wide frequency range of modes in the two $\beta$ Cep stars $\nu$ Eri and 12 Lac. Perhaps there is a need for further refining stellar opacity data. The data from the Independent Project (Seaton 2005, for the latest version), though confirming the gross features of the Z-bump, led to somewhat different astrophysical predictions (e.g. Daszyńska-Daszkiewicz et al. 2005). The interpretation of frequency spectra of Be stars is complicated by the large effects of rotation. Contradicting results of modelling the rich oscillation spectrum of HD 163868 might suggest we still need improvement in our treatment of linear oscillations in the case of fast rotation or again for improving the opacity data.

The mechanism responsible for Be star activity varying on long time scales is still unknown. It is interesting to enquire about the possible role of the opacity driven oscillation. The answer requires going beyond linear theory. There are more such questions regarding $B$ stars, for instance, what determines the main peak amplitude and its harmonics, or what causes temporal amplitude changes seen in many $\beta$ Cep stars. Unfortunately, nonlinear modelling of B-star pulsation is still in its infancy phase. All modelling done so far assumed spherical symmetry. The lesson we learnt from recent studies of radial pulsation is that the detected modes cannot saturate the instability and thus they must represent only a small subset of the excited modes. What is the rest of the modes? Linear stability calculations show that there are many unstable high- $\ell$ modes, which may contribute to saturation but not to the observed variability. However, the presence of such modes should manifest itself in spectral line broadening which cannot be easily hidden. However, a quantitative assessment of this effect is still ahead of us.

Acknowledgments. I am grateful to the SOC for the invitation to this workshop. My participation was supported by the HELAS project. The preparation of my presentation and of this paper was supported by the Polish MNil grant No. 1 P03D 02128. 


\section{References}

Ausseloos M., Scuflaire R., Thoul A., Aerts C., 2004, MNRAS, 355, 352

Aerts C., de Cat P., Handler G., et al., 2004, MNRAS, 347, 463

Baade D., 1982, A\&A, 105, 65

Balona L. A., 1995, MNRAS, 306, 407

Bourge P.-O., Théado S., Thoul A., 2007, Comm. Asteroseis., 150, 203

Charpinet S., Fontaine G., Brassard P., Dorman B., 1996, ApJ, 471, L103

Cox A. N., Morgan S. M., Rogers F. J., Iglesias C. A., 1992, ApJ, 393, 272

Daszyńska-Daszkiewicz J., Dziembowski W. A., Pamyatnykh A. A., 2005, A\&A, 441, 641

De Ridder J., Telting J. H., Balona L. A., et al., 2004, MNRAS, 351, 324

Dziembowski W. A., Pamyatnykh A. A., 1993, MNRAS, 262, 204

Dziembowski W. A., Daszyńska-Daszkiewicz J., Pamyatnykh A. A., 2007, Comm. Asteroseis., 150, 213

Dziembowski W. A., Moskalik P., Pamyatnykh A. A., 1993, MNRAS, 265, 588

Fontaine G., Brassard P., Charpinet S., et al., 2003, ApJ, 597, 518

Gautschy A., Saio H., 1993, MNRAS, 262, 213

Handler G., Shobbrook R. R., Jerzykiewicz M., et al., 2004, MNRAS 347, 454

Handler G., Jerzykiewicz M., Rodríguez E., et al., 2006, MNRAS, 365, 327

Jerzykiewicz M., Handler G., Shobbrook R. R., et al., 2005, MNRAS, 360, 619

Iglesias C. A., Rogers F. J., Wilson B. G., 1992, ApJ, 397, 717

Kiriakidis M., El Eid M. F., Glatzel W., 1992, MNRAS, 255, 1P

Lee U., Saio H., 1989, MNRAS, 237, 875

Moskalik P., Dziembowski W. A., 1992, A\&A, 256, L5

Pamyatnykh A. A., Handler G., Dziembowski W. A., 2004, MNRAS, 350, 1022

Pigulski A., 2007, Comm. Asteroseis., 150, 159

Seaton M., 1999, MNRAS, 307, 1008

Seaton M., 2005, MNRAS, 362, L1

Simon N. R., 1982, ApJ, 260, L87

Smith M. A., 1977, ApJ, 215, 574

Smolec R., Moskalik P., 2007, Comm. Asteroseis., 150, 205

Turcotte S., Richard O., 2005, in Alecian G., Richard O., Vauclair S., eds, Element Stratification in Stars: 40 Years of Atomic Diffusion. EAS Pub. Ser., Vol. 17, EDP Sciences, Les Ulis, p. 357

Townsend R. H. D., 2005, MNRAS, 360, 465

Walker G. A. H., Kuschnig R., Matthews J. M., et al., 2005, ApJ, 635, L77

Waelkens C., 1991, A\&A, 246, 453 